(C) 2018 IEEE. Personal use of this material is permitted. Permission from IEEE must be obtained for all other uses, in any current or future media, including reprinting/republishing this material for advertising or promotional purposes, creating new collective works, for resale or redistribution to servers or lists, or reuse of any copyrighted component of this work in other works

This is the accepted manuscript of: Alessandro Mingotti, Lorenzo Peretto, Roberto Tinarelli, " A novel equivalent power network impedance approach for assessing the time reference in asynchronous measurements" 2017 IEEE International Instrumentation and Measurement Technology Conference (I2MTC), Torino; Italy, 2017, pp. 1-6, doi: 10.1109/I2MTC.2017.7969760 


\title{
A Novel Equivalent Power Network Impedance Approach for Assessing the Time Reference in Asynchronous Measurements
}

\author{
Alessandro Mingotti, Lorenzo Peretto, Roberto Tinarelli \\ Department of Electrical, Electronic and Information Engineering "G. Marconi” \\ Alma Mater Studiorum - University of Bologna \\ Bologna, Italy
}

\begin{abstract}
The paper presents a novel mathematical approach for assessing the phase angle displacement of voltages at nods of a power network with respect to a node taken as reference. It is accomplished without the use of a global time-reference signal, like those provided by GPS technology or through wired connections. This study origins from the growing interest of Distribution System Operators (DSOs) to deploy Distributed Synchronized Measurement Systems (DSMS) in their power networks (like, in particular, the Phasor Measurement Units) for enhancing the stability and reliability of the networks. There are circumstances where bringing a time reference signal to remote PMUs is a difficult task. The paper provides an alternative solution to evaluate phasors in Distribution lines with no need of a global time reference.
\end{abstract}

Keywords-phase angle measurement; time synchronization; network impedance; phase difference; asynchronous measurement; PMU

\section{INTRODUCTION}

With the huge and fast development of Smart Grids and Distributed Generation, the need to perform measurements in many different nodes of the power networks has become of paramount importance for DSO to allow an effective control of the network operation. Furthermore, the possibility to also synchronize the measurements performed at different nodes of the power networks has allowed even to improve the control performance: better control of the operation frequency, fault detection and location, higher network stability, islanding detection and operation, improving the power flow in the network, etc...

As well known, the devices that allow to perform synchronized measurements in the network are referred to as Phasor Measurements Units (PMU) [1]. They allow not only to perform the measurement of the RMS value of the voltages and currents but also of their phases with respect to a global time reference. This way, according to the definition of phasor given by Charles Proteus Steinmetz [2] in 1893, the phasor of a voltage or of a current is given by its RMS value and by its phase difference with respect to a defined time reference. The comparison between the phases of all voltages in a power network allow to evaluate the state estimation of the network, which, in turn, represents the gate for getting the observability of the whole network. So, the added value of a PMU with respect to a typical power meter is given by the possibility to evaluate the phase difference of all voltages in a network through the use of a global time reference.

The use of PMUs in Transmission lines started around 1988 and its usefulness for a better network control and monitoring was well recognized. In Transmission lines the errors allowed in the evaluation of phases is not a critical parameter due to the very long distances and then to the large difference of the voltage phases (in the order of tens of $\mathrm{mrad} / \mathrm{km}$ ). So traditional Voltage Transformer (VT) with 0.2 accuracy class, used for billing purposes, results well suitable for such an application.

However, in Distribution networks this is not going to happen. Distribution lines are far shorter than Transmission lines and the difference between the node voltage phases results often very small, in the order of few $\mathrm{mrad} / \mathrm{km}$. So VT with typical 0.5 accuracy class already installed for billing purposes or measurement in general are no longer suitable for PMU usage. So, besides the need to have an accurate time reference (with standard deviation in the order of $1 \mu$ s or lower) also very accurate voltage transformers are required for assuring a properly accurate evaluation of the voltage phasors.

Nowadays the global time reference can be provided to all PMUs deployed in the network by means of wireless or wired communication protocols. The pulse-per-second (pps) information included in the Global Positioning System (GPS) signals represents the worldwide most used time reference information. It can be easily and freely read by means of antennas and receivers for triggering all PMUs to a unique reference. Among many advantages in using such a technique, it shows, on the contrary, some criticalities: the most important one is represented by the need to install the antenna such that to be able to receive satellite signals. But this is not always occurring in many circumstances, like, for instance, in urban areas, where many obstacles can make it difficult: trees, buildings, skyscrapers, underground secondary substations, roads and others.

In the last decade also wired time reference infrastructures have been developed. Today their performance (in terms of delays and accuracy of the time reference) are getting more and more close to the GPS one. In particular, IEEE 1588 [3] and IEC 61850-5 [4] Standards are by far the most used recommend practices for the transmission of reference times over wired communication infrastructures. However, also such a technology shows some limitations. In particular, in case of a 
lack of a suitable communication network, huge investments are required for its implementation. Moreover, in rural areas the deployment of a wiring infrastructure can result almost impossible.

At the light of the aforementioned issues, a novel analytical method for assessing the phase difference between the voltages at different nodes of a distribution power network is proposed. The main features of such a method are that no global time reference is required for the units deployed in the field and that the accuracy in the measurement of the phase difference of the voltages at nodes is equivalent to that gauged by using existent technologies. It only requires that voltages and currents in each node of the networks be simultaneously acquired with high accuracy. However, the measurements at different nodes are performed asynchronously.

It can be stated that the required very high accuracy for the time stamp measurement in the present PMUs is now turned into the requirement of having very high accuracy in the amplitude measurements. In other words, high accuracy requirement in the time domain has been moved into high accuracy requirement in the amplitude domain.

In the scientific literature, only one application of unsynchronized measurement of phasors in power networks has been found [5]. In that paper, the authors iteratively determine the state of the network formulated by means of the so-called augmented matrix approach. On the contrary, several papers (see e.g. [6-14]) exploit unsynchronized measurements to tackle fault location issues.

The paper is structured as follows: in Section II the method is presented; in Section III a numerical example is given in order to show the performance of the proposed technique; in Section IV comments and conclusions are drown along with the presentation of future work on this topic.

\section{The PROPOSED APPROACH}

\section{A. Theoretical background: Electric line modeling}

Let us briefly recall how an electric line is modeled. As it is well known, an elementary portion of length $d l$ of a single-wire line is usually represented as shown in Fig. 1, where $r, l, c$ and $g$ are the resistance, inductance, capacitance and conductance per unit length, respectively.

The line equations are:

$$
\begin{aligned}
& \frac{d \bar{V}}{d x}=-\bar{z} \bar{I} \\
& \frac{d \bar{I}}{d x}=-\bar{y} \bar{V}
\end{aligned}
$$

where $\bar{V}$ and $\bar{I}$ are the phasor of voltage and current, respectively, $x$ is the distance and:

$$
\begin{gathered}
\bar{z}=r+j \omega l \\
\bar{y}=g+j \omega c,
\end{gathered}
$$

being $\omega$ the rated angular frequency.

For medium voltage cable lines, the effect of $g$ is usually negligible.

By deriving (1) and (2) versus $x$, the following $2^{\text {nd }}$ order differential equations are obtained:

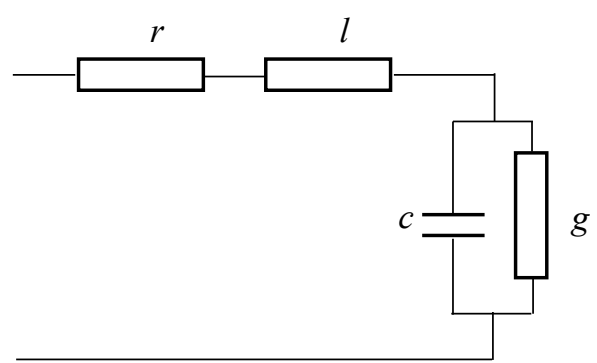

Fig. 1. Model of an elementary portion of a single-wire line

$$
\begin{aligned}
& \frac{d^{2} \bar{V}}{d x^{2}}=\bar{z} \bar{y} \bar{V} \\
& \frac{d^{2} \bar{I}}{d x^{2}}=\bar{z} \bar{y} \bar{I}
\end{aligned}
$$

By defining the propagation constant $\bar{\gamma}$, the characteristic impedance $\overline{Z_{c}}$ and the characteristic admittance $\bar{Y}_{c}$ as in the follows:

$$
\begin{aligned}
& \bar{\gamma}=\sqrt{\bar{z} \bar{y}} \\
& \overline{Z_{c}}=\sqrt{\frac{\bar{z}}{\bar{y}}} \\
& \bar{Y}_{c}=\sqrt{\frac{\bar{y}}{\bar{z}}},
\end{aligned}
$$

the solutions of (5) and (6) can be written as:

$$
\begin{gathered}
\bar{V}=\bar{V}_{1} \cosh (\bar{\gamma} x)-\bar{Z}_{c} \bar{I}_{1} \sinh (\bar{\gamma} x) \\
\bar{I}=-\bar{Y}_{c} \bar{V}_{1} \sinh (\bar{\gamma} x)+\bar{I}_{1} \cosh (\bar{\gamma} x),
\end{gathered}
$$

where $\bar{V}_{1}$ and $\bar{I}_{1}$ are the voltage and current phasors at the beginning of the line $(x=0)$.

Equations (10) and (11) suggest that a distributed parameters single-wire line of given length $\ell$ can be represented by means of a two-port model defined by the following equation:

$$
\left[\begin{array}{l}
\bar{V}_{2} \\
\bar{I}_{2}
\end{array}\right]=\left[\begin{array}{cc}
\bar{A} & -\bar{B} \\
-\bar{C} & \bar{A}
\end{array}\right]\left[\begin{array}{l}
\bar{V}_{1} \\
\bar{I}_{1}
\end{array}\right]
$$

where $\bar{V}_{2}$ and $\bar{I}_{2}$ are the voltage and current phasors at the end of the line $(x=\ell)$ and:

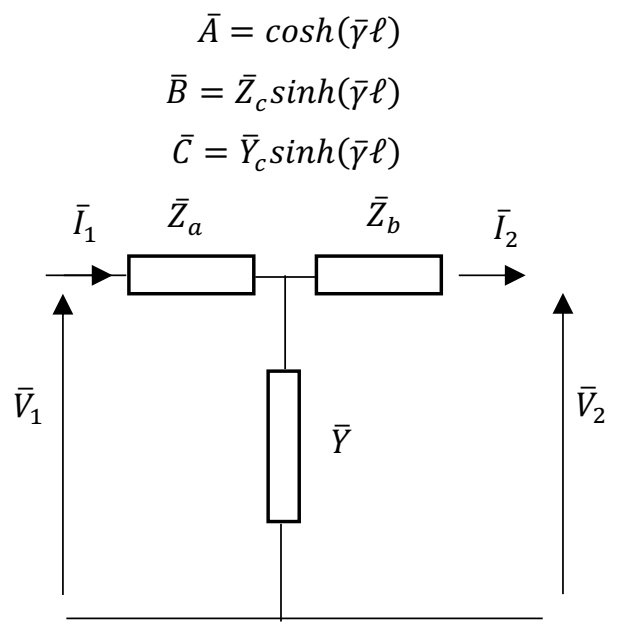

Fig. 2. T-circuit representation of single-wire line 
Therefore, the line can be modeled by means of a $\mathrm{T}$ circuit (Fig. 2) as well as a $\prod$ one. In the former case, which is the representation used in this study, the impedances depicted in Fig. 2 have the following expressions:

$$
\begin{gathered}
\bar{Z}_{a}=\bar{Z}_{b}=\frac{\bar{A}-1}{\bar{C}} \\
\bar{Y}=\bar{C}
\end{gathered}
$$

\section{B. Procedure}

As mentioned in Section I, the main goal of this research is to estimate the phase difference between the voltage $\bar{V}_{1}$ at the beginning of the line, taken as reference and the voltage $\bar{V}_{2}$ at the end of the line without using synchronized measurements (Fig.3).

Such phase difference is due to the combined effect of both the line (according to its model shown in Fig. 2) and the equivalent impedance $\bar{Z}_{L}$ of the load connected at the end of line. The lack of synchronization does not allow to write a sufficient number of independent equations to determine all the three unknown parameters $\left(\bar{Z}_{L}, \bar{Z}_{a}=\bar{Z}_{b}, \bar{Y}\right)$ of such a circuit. Therefore, the proposed method relies on the use of a different model, shown in Fig. 4, whose parameters are computed in a such a way that line losses as well as the active powers at nodes 1 and 2 are the same as the actual ones. As a matter of fact, active power is an integral quantity computed over one (or more) cycles and its value at such nodes are independent of the synchronization of its measurements. Of course, this holds only if the power system is in steady-state conditions for just one cycle in each node.

In the following the procedure for estimating the searched phase difference is described. To this purpose, let us denote by $\Delta \varphi_{\mathrm{a}}$ the actual value of such a phase difference and by $\Delta \varphi_{\mathrm{e}}$ the corresponding estimate provided by the approach.

First of all, the couples of phasors $\bar{V}_{1}$ and $\bar{I}_{1}, \bar{V}_{2}$ and $\bar{I}_{2}$ must be measured. In each measurement node, voltage and current are simultaneously acquired, so that the relationship between the phasors of each couple is correct. Of course, due to the lack of synchronization, the phase difference between $\bar{V}_{1}$ and $\bar{V}_{2}$ is $\Delta \varphi_{\mathrm{a}}$ $+\delta$, where $\delta$ is a random angle depending on the random time difference between the acquisition of the two couples of phasors.

With reference to the circuit in Fig. 4, the following system of equations can be written:

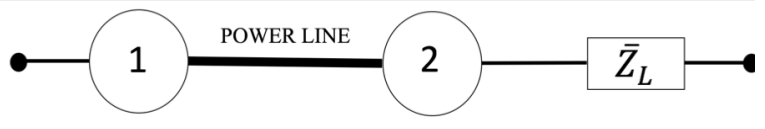

Fig. 3. Single-wire line topology

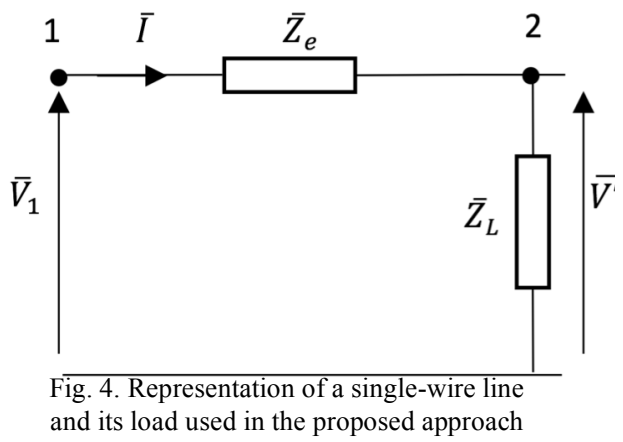

$$
\left\{\begin{array}{c}
P_{2}^{\prime}=R e\left(\frac{\left|\bar{V}_{2}^{\prime}\right|^{2}}{\bar{Z}_{L}^{*}}\right) \\
\bar{V}_{2}^{\prime}=\bar{V}_{1} \frac{\bar{Z}_{L}}{\bar{Z}_{L}+\bar{Z}_{e}}
\end{array}\right.
$$

where $P_{2}^{\prime}$ is the active power at the node 2 of such a circuit. The requirements about the equivalence in terms of active power between the circuit of Fig. 2 and Fig. 4 leads, as stated above, to $P_{2}^{\prime}=P_{2}$, which is known from the measurements of $\bar{V}_{2}$ and $\bar{I}_{2}$. The above measurements allow also to determine the actual value of $\bar{Z}_{L}$ :

$$
\bar{Z}_{L}=\frac{\bar{V}_{2}}{\bar{I}_{2}}
$$

Under the assumption of same line losses in the actual as well as in the proposed circuit, the resistive part $R_{e}$ of the equivalent impedance $\bar{Z}_{e}$ of Fig. 4 can be easily determined:

$$
R_{e}=2 \frac{P_{1}-P_{2}}{I_{1}^{2}+I_{2}^{2}}
$$

where $P_{1}$ and $P_{2}$ are the active powers measured at nodes 1 and 2 , respectively and $I_{1}$ and $I_{2}$ are the RMS values of the above defined current phasors.

Now the system (18) can be solved to find the reactive part $X_{e}$ of $\bar{Z}_{e}$. Some manipulations, reported in appendix, lead to the following $2^{\text {nd }}$ order equation:

$$
X_{e}^{2}+2 X_{e} X_{L}+d=0
$$

where $X_{L}$ is the reactive part of $\bar{Z}_{L}$ and:

$$
d=\left|\bar{Z}_{L}\right|^{2}-\frac{\left|\bar{V}_{1}\right|^{2}\left|\bar{Z}_{L}\right|^{2}}{P_{2}} \operatorname{Re}\left\{\frac{1}{\bar{z}_{L}^{*}}\right\}+R_{e} \bar{Z}_{L}^{*}+R_{e} \bar{Z}_{L}+R_{e}{ }^{2}
$$

One of the solutions of (21) is always negative if, as it is usual, the power factor of $\bar{Z}_{L}$ is lagging.

Finally, $\bar{V}_{2}^{\prime}$ can be determined along with the phase difference $\Delta \varphi_{\mathrm{e}}$, with respect to $\bar{V}_{1}$ by means of the second equation of (18). Fig. 5 summarizes the above described procedure.

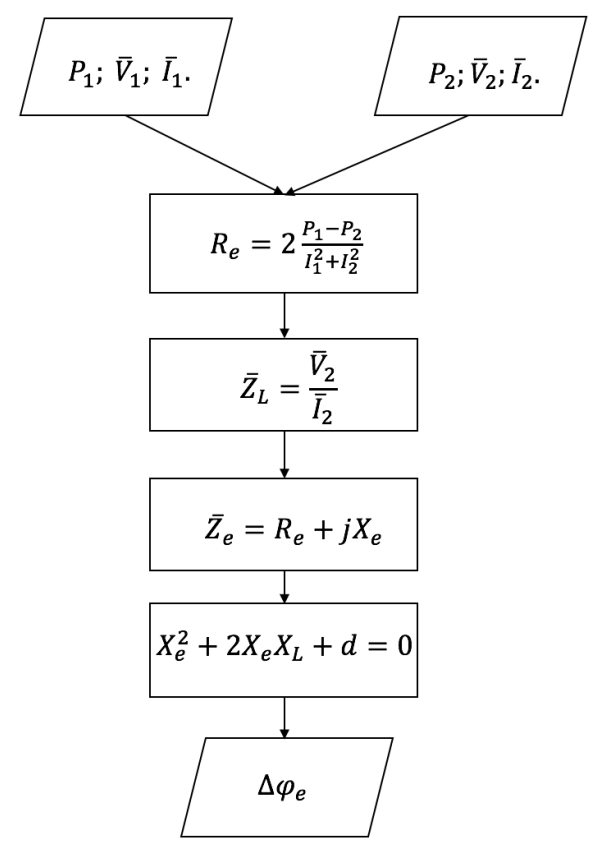

Fig. 5. Procedure for estimating the phase difference $\Delta \varphi_{\mathrm{e}}$ between $\bar{V}_{1}$ and $\bar{V}_{2}^{\prime}$ 


\section{NUMERICAL EXAMPLE}

A numerical example of the proposed procedure is described. To this purpose, three typical medium voltage single-wire cables with different cross sections $\left(50 \mathrm{~mm}^{2}, 95 \mathrm{~mm}^{2}\right.$, and 240 $\mathrm{mm}^{2}$ ) have been chosen. Each cable features the per unit length parameters shown in Table I, where $r, c$, and $l$ have the same meaning as in Section II.

Different cable lengths, representative of typical distance between medium voltage nodes $(5000 \mathrm{~m}, 7500 \mathrm{~m}$, and 10000 $\mathrm{m})$, have been taken into account.

For each combination of cable cross section and length, the equivalent T-circuit of Fig. 2 has been solved by considering different loads. In particular, all the loads have been given 0.8 power factor and apparent power $S_{L}$ ranging from $500 \mathrm{kVA}$ to 8 MVA. The specific values are reported in the following Tables. The solution of such a circuit provided the reference phase difference $\Delta \varphi_{a}$ between $\bar{V}_{1}$ and $\bar{V}_{2}$.

TABLE I. PER UNIT LENGTH PARAMETERS FOR THE THREE TYPES OF CABLE.

\begin{tabular}{|c|c|c|c|}
\hline $\mathbf{S}\left(\mathbf{m m}^{\mathbf{2}}\right)$ & $\mathbf{r}(\mathbf{m} \boldsymbol{\Omega} / \mathbf{m})$ & $\mathbf{l}(\mathbf{u H} / \mathbf{m})$ & $\mathbf{c ~ ( n F / m ) ~}$ \\
\hline $\mathbf{5 0}$ & 0.587 & 0.4138 & 0.21 \\
\hline $\mathbf{9 5}$ & 0.193 & 0.3694 & 0.26 \\
\hline $\mathbf{2 4 0}$ & 0.0754 & 0.324 & 0.37 \\
\hline
\end{tabular}

The proposed procedure has been then applied to the data obtained from the above simulations to estimate the phase difference $\Delta \varphi_{e}$ between $\bar{V}_{1}$ and $\bar{V}_{2}$.

Tables II, III and IV show the results for different lengths when the cable section is $50 \mathrm{~mm}^{2}$. Table V, VI and VII refer to the $95 \mathrm{~mm}^{2}$ case and, lastly, Table VIII, IX and X deal with the $240 \mathrm{~mm}^{2}$ cable.

TABLE II. ACTUAL AND ESTIMATED VOLTAGE PHASE DIFFERENCE FOR DIFFERENT LOADS IN A 5000 M LENGTH, 50 $\mathrm{MM}^{2}$ SECTION CABLE.

\begin{tabular}{|c|c|c|c|}
\hline $\mathbf{S}_{\mathrm{L}}(\mathbf{k V A})$ & $\Delta \varphi_{\mathrm{a}}(\mathbf{m r a d})$ & $\Delta \varphi_{\mathrm{e}}(\mathbf{m r a d})$ & $\Delta \varphi_{\mathrm{a}}-\Delta \varphi_{\mathrm{e}}(\mathbf{m r a d})$ \\
\hline 500 & 2.06 & 2.53 & 0.46 \\
\hline 1000 & 4.42 & 4.88 & 0.45 \\
\hline 1500 & 6.74 & 7.20 & 0.45 \\
\hline 2000 & 9.03 & 9.48 & 0.45 \\
\hline 4000 & 17.86 & 18.32 & 0.45 \\
\hline 8000 & 34.14 & 34.59 & 0.44 \\
\hline
\end{tabular}

TABLE III. ACTUAL AND ESTIMATED VOLTAGE PHASE DIFFERENCE FOR DIFFERENT LOADS IN A 7500M LENGTH, $50 \mathrm{MM}^{2}$ SECTION CABLE.

\begin{tabular}{|c|c|c|c|}
\hline $\mathbf{S}_{\mathrm{L}}(\mathbf{k V A})$ & $\Delta \varphi_{\mathrm{a}}(\mathbf{m r a d})$ & $\Delta \varphi_{\mathrm{e}}(\mathbf{m r a d})$ & $\Delta \varphi_{\mathrm{a}}-\Delta \varphi_{\mathrm{e}}$ (mrad) \\
\hline 500 & 2.85 & 3.89 & 1.03 \\
\hline 1000 & 6.35 & 7.38 & 1.03 \\
\hline 1500 & 9.77 & 10.80 & 1.03 \\
\hline 2000 & 13.12 & 14.15 & 1.03 \\
\hline 4000 & 25.84 & 26.86 & 1.02 \\
\hline 8000 & 48.44 & 49.44 & 1.00 \\
\hline
\end{tabular}

TABLE IV. ACTUAL AND ESTIMATED VOLTAGE PHASE DIFFERENCE FOR DIFFERENT LOADS IN A 10000 M LENGTH, $50 \mathrm{MM}^{2}$ SECTION CABLE.

\begin{tabular}{|c|c|c|c|}
\hline $\mathbf{S}_{\mathbf{L}}(\mathbf{k V A})$ & $\Delta \varphi_{\mathbf{a}}(\mathbf{m r a d})$ & $\Delta \varphi_{\mathrm{e}}(\mathbf{m r a d})$ & $\Delta \boldsymbol{\varphi}_{\mathrm{a}}-\Delta \boldsymbol{\varphi}_{\mathrm{e}}(\mathbf{m r a d})$ \\
\hline 500 & 3.47 & 5.31 & 1.84 \\
\hline 1000 & 8.09 & 9.93 & 1.83 \\
\hline 1500 & 12.58 & 14.41 & 1.83 \\
\hline 2000 & 16.94 & 18.77 & 1.82 \\
\hline 4000 & 33.26 & 35.07 & 1.81 \\
\hline 8000 & 61.22 & 63.00 & 1.78 \\
\hline
\end{tabular}

TABLE V. ACTUAL AND ESTIMATED VOLTAGE PHASE DIFFERENCE FOR DIFFERENT LOADS IN A 5000M LENGTH, 95 $\mathrm{MM}^{2}$ SECTION CABLE.

\begin{tabular}{|c|c|c|c|}
\hline $\mathbf{S}_{\mathbf{L}}(\mathbf{k V A})$ & $\Delta \varphi_{\mathrm{a}}(\mathbf{m r a d})$ & $\Delta \varphi_{\mathrm{e}}(\mathbf{m r a d})$ & $\Delta \varphi_{\mathrm{a}}-\Delta \varphi_{\mathrm{e}}(\mathbf{m r a d})$ \\
\hline 500 & 0.23 & 0.58 & 0.35 \\
\hline 1000 & 0.65 & 1.00 & 0.35 \\
\hline 1500 & 1.08 & 1.43 & 0.35 \\
\hline 2000 & 1.49 & 1.85 & 0.35 \\
\hline 4000 & 3.13 & 3.48 & 0.34 \\
\hline 8000 & 6.26 & 6.60 & 0.34 \\
\hline
\end{tabular}

TABLE VI. ACTUAL AND ESTIMATED VOLTAGE PHASE DIFFERENCE FOR DIFFERENT LOADS IN A 7500 M LENGTH, 95 $\mathrm{MM}^{2}$ SECTION CABLE.

\begin{tabular}{|c|c|c|c|}
\hline $\mathbf{S}_{\mathrm{L}}(\mathbf{k V A})$ & $\Delta \varphi_{\mathrm{a}}(\mathbf{m r a d})$ & $\Delta \varphi_{\mathrm{e}}(\mathbf{m r a d})$ & $\Delta \varphi_{\mathrm{a}}-\Delta \varphi_{\mathrm{e}}$ (mrad) \\
\hline 500 & 0.20 & 0.99 & 0.79 \\
\hline 1000 & 0.83 & 1.62 & 0.79 \\
\hline 1500 & 1.46 & 2.25 & 0.79 \\
\hline 2000 & 2.08 & 2.87 & 0.79 \\
\hline 4000 & 4.48 & 5.27 & 0.78 \\
\hline 8000 & 8.97 & 9.73 & 0.76 \\
\hline
\end{tabular}

TABLE VII. ACTUAL AND ESTIMATED VOLTAGE PHASE DIFFERENCE FOR DIFFERENT LOADS IN A 10000 M LENGTH, $95 \mathrm{MM}^{2}$ SECTION CABLE.

\begin{tabular}{|c|c|c|c|}
\hline $\mathrm{S}_{\mathrm{L}}(\mathrm{kVA})$ & $\Delta \varphi_{\mathrm{a}}(\mathbf{m r a d})$ & $\Delta \varphi_{\mathrm{e}}(\mathbf{m r a d})$ & $\Delta \varphi_{\mathrm{a}}-\Delta \varphi_{\mathrm{e}}(\mathbf{m r a d})$ \\
\hline 500 & 0.69 & 1.485 & 1.415 \\
\hline 1000 & 0.914 & 2.325 & 1.411 \\
\hline 1500 & 1.744 & 3.151 & 1.406 \\
\hline 2000 & 2.561 & 3.963 & 1.402 \\
\hline 4000 & 5.700 & 7.084 & 1.384 \\
\hline 8000 & 11.420 & 12.771 & 1.351 \\
\hline
\end{tabular}

TABLE VIII. ACTUAL AND EXTRACTED VOLTAGE PHASE DIFFERENCE FOR DIFFERENT LOADS IN A 5000 M LENGTH, $240 \mathrm{MM}^{2}$ SECTION CABLE.

\begin{tabular}{|c|c|c|c|}
\hline $\mathbf{S}_{\mathbf{L}}(\mathbf{k V A})$ & $\Delta \boldsymbol{\varphi}_{\mathrm{a}}(\mathbf{m r a d})$ & $\Delta \varphi_{\mathrm{e}}(\mathbf{m r a d})$ & $\Delta \varphi_{\mathrm{a}}-\Delta \varphi_{\mathrm{e}}(\mathbf{m r a d})$ \\
\hline 500 & -0.77 & -0.46 & 0.30 \\
\hline 1000 & -1.43 & -1.13 & 0.30 \\
\hline 1500 & -2.09 & -1.43 & 0.30 \\
\hline 2000 & -2.75 & -2.44 & 0.30 \\
\hline 4000 & -5.34 & -5.04 & 0.29 \\
\hline 8000 & -10.39 & -10.10 & 0.28 \\
\hline
\end{tabular}


TABLE IX. ACTUAL AND ESTIMATED VOLTAGE PHASE DIFFERENCE FOR DIFFERENT LOADS IN A 7500 M LENGTH, $240 \mathrm{MM}^{2}$ SECTION CABLE.

\begin{tabular}{|c|c|c|c|}
\hline $\mathbf{S}_{\mathbf{L}}(\mathbf{k V A})$ & $\Delta \varphi_{\mathrm{a}}(\mathbf{m r a d})$ & $\Delta \varphi_{\mathrm{e}}(\mathbf{m r a d})$ & $\Delta \varphi_{\mathrm{a}}-\Delta \varphi_{\mathrm{e}}(\mathbf{m r a d})$ \\
\hline 500 & -1.24 & -0.55 & 0.68 \\
\hline 1000 & -2.23 & -1.54 & 0.68 \\
\hline 1500 & -3.21 & -2.53 & 0.67 \\
\hline 2000 & -4.18 & -3.51 & 0.67 \\
\hline 4000 & -8.02 & -7.35 & 0.67 \\
\hline 8000 & -15.40 & -14.75 & 0.65 \\
\hline
\end{tabular}

TABLE $\mathrm{X}$. ACTUAL AND ESTIMATED VOLTAGE PHASE DIFFERENCE FOR DIFFERENT LOADS IN A 10000 M LENGTH, $240 \mathrm{MM}^{2}$ SECTION CABLE.

\begin{tabular}{|c|c|c|c|}
\hline $\mathbf{S}_{\mathbf{L}}(\mathbf{k V A})$ & $\Delta \varphi_{\mathrm{a}}(\mathbf{m r a d})$ & $\Delta \varphi_{\mathrm{e}}(\mathbf{m r a d})$ & $\Delta \varphi_{\mathrm{a}}-\Delta \varphi_{\mathrm{e}}(\mathbf{m r a d})$ \\
\hline 500 & -1.76 & -0.54 & 1.21 \\
\hline 1000 & -3.07 & -1.86 & 1.21 \\
\hline 1500 & -4.38 & -3.17 & 1.20 \\
\hline 2000 & -5.67 & -4.46 & 1.20 \\
\hline 4000 & -10.71 & -9.54 & 1.17 \\
\hline 8000 & -20.30 & -19.16 & 1.14 \\
\hline
\end{tabular}

As it is expected, the longer is the cables the larger is the phase difference between the phasors measured at the two nodes of the line. This fact is confirmed also by the results. In fact, as can be seen for the $50 \mathrm{~mm}^{2}$ cable in Tables II, III, and IV, $\Delta \varphi_{a}$ values with a $500 \mathrm{kVA}$ load are $2.06,2.85,3.47 \mathrm{mrad}$, for a $5000,7500,10000 \mathrm{~m}$ cable, respectively. In the same tables also the estimated phase difference is presented. In the right-hand column the estimation error is shown. The absolute value of such an error is almost constant versus the load value. the larger is the line length the larger is the error. Same consideration applies for the other line cross sections. In case of 2 MVA load and $7500 \mathrm{~m}$ cable, for all the three sections, Tables III, VI, and IX show that the error decreases with the increase of the cable cross section. In particular, it is $1.03,0.79,0.67 \mathrm{mrad}$ for a $50,95,240 \mathrm{~mm}^{2}$ cable, respectively. It can be concluded that the proposed procedure features better performance in case of shorter lines, higher cross section and in presence of high loaded lines. It must be highlighted that such errors result well acceptable for PMU applications. In fact, typical actual phase displacements in the DSMS systems are in the order of few mrad.

\section{Final Remarks AND Future Work}

Numerical results have shown the effectiveness of the proposed approach for evaluating the phase difference between voltages at different nodes of a power network. It must be highlighted that according to the presented approach the measurement of the voltage phasors can be accomplished by using power and energy meters. The major advantage of the proposed procedure and architecture is that measurements can be performed asynchronously and without using a global time reference. It reflects into a simpler and less expensive measurement architecture. This way a larger deployment of instrumentation can be scheduled with benefits in term of enhanced network observability (mainly in presence of DG), redundancy and, finally, more accurate measurements. The need of highly accurate measurements of time has been now converted into the requirement of having very high accuracy in the amplitude measurements. High accuracy requirement in the time domain has been then moved now into two main requirements: i) high accuracy in amplitude measurements and ii) narrow time-skew between voltage and current measurements of each energy meter. So accuracy and, then, uncertainty has now all related to the measuring instrument; no more influence in the phase difference measurements will be given by the latency in the time reference transmission.

One of main requirements for the presented approach is that during the period of time in which measurements at all nodes are performed, the system should be assumed under steady state conditions. However, this constraint is not particularly heavy for the method as the measurements at all nodes can, for instance, be performed starting from the zero crossing of the voltages. In this way all measurements will be performed in the same period of time in all nodes. The only difference in time is represented by the time delay due to the network topology (line impedances and loads). In particular in this way the method will not be affected by the very actual phenomenon represented by the change of network frequency over time [14], as all expressions would be evaluated for all nodes under the same operating conditions. So also one of the most useful parameters measured by PMU for the identification of the network stability [1], the Rate Of Change Of Frequency (ROCOF), will be measured by means of the presented approach.

However, it should be kept in mind that accuracy in measurement of electrical quantities (voltages and currents) represents the most important requirements for this method for properly working. As underlined in the Section I, the uncertainty in the measurements turns into an uncertainty in the phase difference between voltages.

In future works authors will deeply investigate on the propagation of uncertainties through the presented algorithm arising from the hardware items used and on its performance in case of lack of observability of the network (i.e. not all nodes of the network equipped with an energy meter).

\section{APPENDIX}

In this Section, the solution of the system (18) in Section II, below rewritten as (23) is shown:

$$
\left\{\begin{array}{c}
P_{2}^{\prime}=\operatorname{Re}\left(\frac{\left|\bar{V}_{2}^{\prime}\right|^{2}}{\bar{Z}_{L}^{*}}\right) \\
\bar{V}_{2}^{\prime}=\bar{V}_{1} \frac{\bar{Z}_{L}}{\bar{Z}_{L}+\bar{Z}_{e}}
\end{array}\right.
$$

As described in the paper and according to Fig. 4, the estimated active power $P_{2}^{\prime}$ must be equal to the actual active power $P_{2}$ measured at the second node of the line, hence:

$$
P_{2}^{\prime}=\operatorname{Re}\left(\frac{\left|\bar{V}_{2}^{\prime}\right|^{2}}{\bar{z}_{L}^{*}}\right)=P_{2}
$$

Remembering that the real part of the product between two complex numbers is:

$$
\operatorname{Re}(\bar{a} \bar{b})=a_{R} b_{R}-a_{I} b_{I}
$$


where the subscripts $R$ and $I$ indicate the real part and the imaginary coefficient of the complex numbers, respectively. By applying the (25) to the (24) and knowing that $\left|\bar{V}_{2}^{\prime}\right|^{2}$ is real:

$$
P_{2}=\operatorname{Re}\left(\frac{1}{\bar{Z}_{L}^{*}}\right) \cdot\left|\bar{V}_{2}^{\prime}\right|^{2}-0
$$

By substituting the second of the (23) into the (26):

$$
P_{2}=\operatorname{Re}\left(\frac{1}{\bar{Z}_{L}^{*}}\right) \cdot\left|\bar{V}_{1} \frac{\bar{Z}_{L}}{\overline{\bar{Z}}_{L}+\bar{Z}_{e}}\right|^{2}=\operatorname{Re}\left(\frac{1}{\overline{\bar{Z}}_{L}^{*}}\right) \cdot \frac{\left|\bar{V}_{1}\right|^{2}\left|\bar{Z}_{L}\right|^{2}}{\left|\bar{Z}_{L}+\bar{Z}_{e}\right|^{2}}
$$

The variable $X_{e}$ is contained into $\bar{Z}_{e}$ and then it must be extracted from the (27):

$$
\left|\bar{Z}_{L}+\bar{Z}_{e}\right|^{2}=\operatorname{Re}\left(\frac{1}{\bar{Z}_{L}^{*}}\right) \cdot \frac{\left|\bar{V}_{1}\right|^{2}\left|\bar{Z}_{L}\right|^{2}}{P_{2}}
$$

Given that all the terms in the right side of (28) are known, for sake of brevity they can be named as:

$$
F=\operatorname{Re}\left(\frac{1}{\bar{Z}_{L}^{*}}\right) \cdot \frac{\left|\bar{V}_{1}\right|^{2}\left|\bar{Z}_{L}\right|^{2}}{P_{2}}
$$

By arranging terms into (28) it holds:

$$
\begin{aligned}
& \left|\bar{Z}_{L}+\bar{Z}_{e}\right|^{2}=\left(\bar{Z}_{L}+\bar{Z}_{e}\right)\left(\bar{Z}_{L}{ }^{*}+\bar{Z}_{e}^{*}\right)= \\
& \quad=\left|\bar{Z}_{L}\right|^{2}+\left|\bar{Z}_{e}\right|^{2}+\bar{Z}_{L} \bar{Z}_{e}{ }^{*}+\bar{Z}_{L}{ }^{*} \bar{Z}_{e}=F
\end{aligned}
$$

Equation (30) does not have a single solution because it contains two variables: $R_{e}$ and $X_{e}$. However, as stated above, the equivalent resistance of the line is evaluated in a previous step; therefore in (29) it is a known term.

$$
\begin{aligned}
& \left|R_{e}+j X_{e}\right|^{2}+\bar{Z}_{L}\left(R_{e}-j X_{e}\right)+\bar{Z}_{L}^{*}\left(R_{e}+j X_{e}\right)+ \\
& +\left|\bar{Z}_{L}\right|^{2}=F
\end{aligned}
$$

After some arrangements:

$$
R_{e}{ }^{2}+X_{e}{ }^{2}-j X_{e}\left(\bar{Z}_{L}-\bar{Z}_{L}^{*}\right)+H=0
$$

where:

$$
H=\left|\bar{Z}_{L}\right|^{2}+\bar{Z}_{L} R_{e}+\bar{Z}_{L}^{*} R_{e}-F
$$
II:

Finally (32) leads to the expression (21) written in Section

$$
X_{e}^{2}+2 X_{e} X_{L}+d=0
$$

Now, with the reduced quadratic equation expression the two solution of the (34) can be obtained:

$$
\begin{aligned}
& X_{e_{1,2}}=-X_{L} \pm \sqrt{X_{L}^{2}-d} \\
& X_{e_{1}}=-X_{L}+\sqrt{X_{L}^{2}-d} \\
& X_{e_{2}}=-X_{L}-\sqrt{X_{L}^{2}-d}
\end{aligned}
$$

From (35a) and (35b) it can be stated that, while the reactive part of a line is inductive, the (35b) is always negative and can be then discarded. This way the solution of the quadratic equation (34) is unique.

\section{REFERENCES}

[1] A. Monti, C. Muscas, F. Ponci, "Phasor Measurement Units and Wide Area Monitoring Systems, From the sensor to the systems", Elsevier, 2016.

[2] Steinmetz CP. Complex quantities and their use in electrical engineering. In: Proceedings of the international electrical congress. AIEE; 1893. p. 33-74.

[3] IEEE, IEEE standard for a precision clock synchronization protocol for networked measure- ment and control systems, IEEE Std 1588-2008 (Revision of IEEE Std 1588-2002), July 2008.

[4] IEC, "Communication networks and systems for power utility automation - Part 5: Communication requirements for functions and device models", IEC Standard IEC 61850-5:2013; 2013.

[5] P. Janssen, T. Sezi, J.C. Maun, "Distribution system state estimation using unsynchronized phasor measurements", Proc. of IEEE 2012 ISGT Europe, pp. 1-6, 2012.

[6] A.L. Dalcastagne, S. Noceti Filho, H.H. Zurn, R. Seara, “An Iterative Two-Terminal Fault-Location Method Based on Unsynchronized Phasors", IEEE Transactions on Power Delivery, Oct. 2008.

[7] D. Novosel , D. G. Hart , E. Udren and J. Garitty, "Unsynchronized twoterminal fault location estimation", IEEE Trans. Power Del., vol. 11, no. 1,pp. 130-138, 1996

[8] A.A. Girgis, D.G. Hart, W.L. Peterson, "Unsynchronized two-terminal fault location estimation", IEEE Transactions on Power Delivery, vol. 7, no.1, Jan 1996.

[9] Chi-Shan Yu; Li-Ren Chang; Jr-Ru Cho, "New Fault Impedance Computations for Unsynchronized Two-Terminal Fault-Location Computations", IEEE Transactions on Power Delivery, Oct. 2011.

[10] J. Izykowski, R. Molag, E. Rosolowski, M.M. Saha, “Accurate location of faults on power transmission lines with use of two-end unsynchronized measurements", IEEE Transactions on Power Delivery, vol. 21, no.2, April 2006.

[11] M. Fulczyk, P. Balcerek, J. Izykowski, E. Rosolowski, M. M. Saha, "Two-end unsynchronized fault location algorithm for double-circuit series compensated lines", Proc. of IEEE Power and Energy Society General Meeting, 2008.

[12] B. Mahamedi, J.G. Zhu, "Unsynchronized Fault Location Based on the Negative-Sequence Voltage Magnitude for Double-Circuit Transmission Lines”, IEEE Transactions on Power Delivery, vol. 29, no.4, Aug. 2014.

[13] L. Yuansheng, W. Gang, L. Haifeng, "Time-Domain Fault-Location Method on HVDC Transmission Lines Under Unsynchronized Two-End Measurement and Uncertain Line Parameters", IEEE Transactions on Power Delivery, vol. 30, no.3, June 2015.

[14] IEC EN 50160 "Voltage characteristics of electricity supplied by public distribution systems". 